\title{
SCALING LIMITS FOR SHORTEST PATH \\ LENGTHS ALONG THE EDGES OF STATIONARY TESSELLATIONS
}

\author{
FLORIAN VOSS, ${ }^{*}$ Ulm University \\ CATHERINE GLOAGUEN, ${ }^{* *}$ Orange Labs \\ VOLKER SCHMIDT, ${ }^{* * *}$ Ulm University
}

\begin{abstract}
We consider spatial stochastic models, which can be applied to, e.g. telecommunication networks with two hierarchy levels. In particular, we consider Cox processes $X_{L}$ and $X_{H}$ concentrated on the edge set $T^{(1)}$ of a random tessellation $T$, where the points $X_{L, n}$ and $X_{H, n}$ of $X_{L}$ and $X_{H}$ can describe the locations of low-level and high-level network components, respectively, and $T^{(1)}$ the underlying infrastructure of the network, such as road systems, railways, etc. Furthermore, each point $X_{L, n}$ of $X_{L}$ is marked with the shortest path along the edges of $T$ to the nearest (in the Euclidean sense) point of $X_{H}$. We investigate the typical shortest path length $C^{*}$ of the resulting marked point process, which is an important characteristic in, e.g. performance analysis and planning of telecommunication networks. In particular, we show that the distribution of $C^{*}$ converges to simple parametric limit distributions if a scaling factor $\kappa$ converges to 0 or $\infty$. This can be used to approximate the density of $C^{*}$ by analytical formulae for a wide range of $\kappa$.
\end{abstract}

Keywords: Stochastic geometry; random geometric graph; Cox process; Palm distribution; Poisson approximation; uniform integrability; subadditive ergodic theorem; Blaschke-Petkantschin formula; telecommunication network

2010 Mathematics Subject Classification: Primary 60D05

Secondary 60G55; 60F99; 90B15

\section{Introduction}

Asymptotic properties of spatial stochastic models are considered, which can be applied in, e.g. the analysis and planning of telecommunication networks. More precisely, we consider stochastic models for networks with two hierarchy levels, i.e. there are network components of two different kinds: low-level components (LLCs) and high-level components (HLCs). The locations of both HLCs and LLCs are represented by points in the Euclidean plane $\mathbb{R}^{2}$. We then associate with each HLC a certain subset of $\mathbb{R}^{2}$ which is called its serving zone. This is done in such a way that the serving zones of the HLCs are disjoint convex polygons which cover the whole of $\mathbb{R}^{2}$. Each LLC is linked to the HLC in whose serving zone the LLC is located. In particular, we assume that the serving zones are constructed as the cells of the Voronoi tessellation with respect to the locations of HLCs. This is equivalent to linking each LLC to its nearest HLC, where 'nearest' means with respect to the Euclidean distance. Furthermore, we

Received 13 May 2009; revision received 23 December 2009.

* Current address: Medical Data Services, Boehringer Ingelheim Pharma GmbH \& Co. KG, Binger Str. 173, 55216

Ingelheim, Germany. Email address: florian.voss@boehringer-ingelheim.com

** Postal address: Orange Labs, 38-40, rue du Général Leclerc, 92794 Issy-les-Moulineaux, France.

*** Postal address: Insitute of Stochastics, Ulm University, Helmholtzstr. 18, 89069 Ulm, Germany. 
assume that HLCs and LLCs are located on the edges of a random geometric graph, where the link from an LLC to its nearest HLC is assumed to be the shortest path along the edges of that graph. In the case of telecommunication networks the edges of the random geometric graph represent the underlying infrastructure, e.g. an inner-city street system.

We thus study a class of stochastic network models which has been introduced in [11] as the stochastic subscriber line model (SSLM) for urban access networks. Note that the SSLM is a model from stochastic geometry which provides tools for the description of geometric features of the network. Based on this model, stochastic econometrical analysis can be done for real telecommunication networks, e.g. connection costs for access networks can be determined (see [10], [14], [30], and [33]), where we focus on the case in which the infrastructure of the network is modeled by the edge set of a stationary random tessellation and both the HLC and LLC are modeled by Cox processes concentrated on this edge set. Then we are especially interested in the shortest path length along the edge set between LLCs and HLCs, which is an important performance characteristic in cost and risk analysis as well as in strategic planning of wired telecommunication. In order to define an appropriately chosen (global) distribution of the shortest path length, we investigate the so-called typical shortest path length $C^{*}$. It can be interpreted as the length of the shortest path from a location of LLCs, which is chosen at random among all locations of LLCs, and its nearest HLC. We are then interested in the asymptotic behavior of the distribution of $C^{*}$ for two extreme cases of model parameters. In particular, we show that the distribution of $C^{*}$ converges to simple parametric limit distributions if a scaling factor $\kappa$ converges to 0 or $\infty$. This can be used to approximate the density of $C^{*}$ by analytical formulae for a wide range of $\kappa$, which is a great advantage, e.g. for the econometrical analysis of real telecommunication networks; see [10]. The mathematical techniques, which we exploit in order to derive our main results presented in Theorems 3.1 and 3.2, include Palm calculus and Poisson approximation for stationary point processes, Kingman's subadditive ergodic theorem, and the generalized Blaschke-Petkantschin formula from geometric measure theory.

The paper is organized as follows. In Section 2 we give a short description of the particular stochastic network model considered in the present paper. Then, in Section 3, we present the main results stated in Theorems 3.1 and 3.2. The proof of Theorem 3.2 is given in Section 4, where some details are postponed to Appendix A. In Section 5, it is shown that the mixing and integrability conditions of Theorems 3.1 and 3.2 are fulfilled for various examples of random tessellations. Finally, in Section 6 we conclude the paper and give an outlook to possible future research. In particular, some extensions of our results to other performance characteristics, more general classes of random geometric graphs, and more general connection rules are discussed. A more detailed version of the present paper can be found in [32].

\section{Stochastic modeling of hierarchical networks}

To begin with, we give a short description of the particular stochastic network model considered in the present paper. Moreover, we briefly introduce the notation we will use. For details on point processes and random tessellations, see, e.g. [7], [8], [25], [26], and [27]. Surveys on applications of tools from stochastic geometry to spatial stochastic modeling of telecommunication networks can be found in, e.g. [16] and [37].

First we recall some basic notions regarding marked point processes in $\mathbb{R}^{2}$. Let $\mathscr{B}^{2}$ denote the family of Borel sets of $\mathbb{R}^{2}$, and let $\mathbb{M}$ be a Polish space with its Borel $\sigma$-algebra $\mathscr{B}_{\mathbb{M}}$. Furthermore, let $X=\left\{\left(X_{n}, M_{n}\right)\right\}$ be a marked point process with mark space $\mathbb{M}$. Then we regard $X$ as a random element of $\left(N_{\mathbb{M}}, \mathcal{N}_{\mathbb{M}}\right)$, where $N_{\mathbb{M}}$ is the family of all counting measures on $\mathcal{B}^{2} \otimes \mathcal{B}_{\mathbb{M}}$ which are simple and locally finite in the first component and $\mathcal{N}_{\mathbb{M}}$ is the usual $\sigma$-algebra 
on $N_{\mathbb{M}}$. For $x \in \mathbb{R}^{2}$, we define the shift operator $t_{x}$ by $t_{x} X=t_{x}\left\{\left(X_{n}, M_{n}\right)\right\}=\left\{\left(X_{n}-x, M_{n}\right)\right\}$. Now assume that $X=\left\{\left(X_{n}, M_{n}\right)\right\}$ is stationary with intensity $\lambda>0$. Then the Palm mark distribution $\mathrm{P}_{X}^{o}: \mathcal{B}_{\mathbb{M}} \rightarrow[0,1]$ of $X$ is given by

$$
\mathrm{P}_{X}^{o}(G)=\frac{1}{\lambda} \mathrm{E} \#\left\{n: X_{n} \in[0,1)^{2}, M_{n} \in G\right\}, \quad G \in \mathcal{B}_{\mathbb{M}} .
$$

A random variable $M^{*}$ distributed according to $\mathrm{P}_{X}^{o}$ is called the typical mark of $X$.

In the following, two jointly stationary marked point processes $X^{(1)}=\left\{\left(X_{n}^{(1)}, M_{n}^{(1)}\right)\right\}$ and $X^{(2)}=\left\{\left(X_{n}^{(2)}, M_{n}^{(2)}\right)\right\}$ with intensities $\lambda_{1}$ and $\lambda_{2}$ and mark spaces $\mathbb{M}_{1}$ and $\mathbb{M}_{2}$, respectively, will be considered as a random element $Y=\left(X^{(1)}, X^{(2)}\right)$ of the product space $N_{\mathbb{M}_{1}, \mathbb{M}_{2}}=$ $N_{\mathbb{M}_{1}} \times N_{\mathbb{M}_{2}}$. The Palm distribution $\mathrm{P}_{X^{(i)}}^{*}$ of $Y$ with respect to the $i$ th component, $i=1,2$, is then defined on $\mathcal{N}_{\mathbb{M}_{1}} \otimes \mathcal{N}_{\mathbb{M}_{2}} \otimes \mathcal{B}_{\mathbb{M}_{i}}$ by

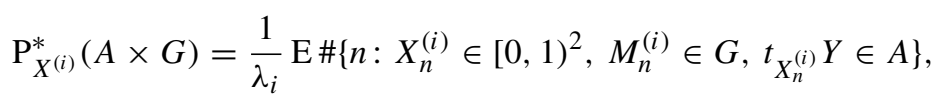

where $A \in \mathcal{N}_{\mathbb{M}_{1}} \otimes \mathcal{N}_{\mathbb{M}_{2}}$ and $G \in \mathscr{B}_{\mathbb{M}_{i}}$. Note that the Palm mark distribution $\mathrm{P}_{X^{(i)}}^{o}$ of $X^{(i)}$ can be obtained from $\mathrm{P}_{X^{(i)}}^{*}$ as a marginal distribution.

As a model for the underlying random geometric graph, we consider the edge set $T^{(1)}$ of a random tessellation $T=\left\{\Xi_{n}\right\}$ of $\mathbb{R}^{2}$. If $T$ is stationary then we define the intensity $\gamma$ of $T$ as $\gamma=\mathrm{E} v_{1}\left(T^{(1)} \cap[0,1]^{2}\right)$, where $v_{1}$ denotes the 1-dimensional Hausdorff measure. In the following we always assume that $T$ is a (normalized) stationary tessellation with $\mathrm{E} \nu_{1}\left(T^{(1)} \cap[0,1]^{2}\right)=1$. Furthermore, for each $\gamma>0$, we consider the scaled tessellation $T_{\gamma}$ with intensity $\gamma$ defined by $T_{\gamma}=T / \gamma$, i.e. we scale the edge set $T^{(1)}$ such that $\mathrm{E} v_{1}\left(T_{\gamma}^{(1)} \cap[0,1]^{2}\right)=\gamma$. Note that a stationary tessellation $T$ is called ergodic and mixing if the random closed set $T^{(1)}$ is ergodic and mixing, respectively.

For any $\gamma>0$, we consider Cox point processes $X_{H}=\left\{X_{H, n}\right\}$ and $X_{L}=\left\{X_{L, n}\right\}$ concentrated on $T_{\gamma}^{(1)}$ in order to model the locations of HLCs and LLCs, respectively. In particular, we assume that $X_{H}$ is a Cox process on $T_{\gamma}^{(1)}$ with linear intensity $\lambda_{\ell}$ which is constructed by placing homogeneous Poisson processes on the edges of $T_{\gamma}$ with linear intensity $\lambda_{\ell}$. Analogously, $X_{L}$ is a Cox process on $T_{\gamma}^{(1)}$ with linear intensity $\lambda_{\ell}^{\prime}$, where we assume that $X_{H}$ and $X_{L}$ are conditionally independent given $T_{\gamma}$. Note that the planar intensities $\lambda$ and $\lambda^{\prime}$ of $X_{H}$ and $X_{L}$ are given by $\lambda=\lambda_{\ell} \gamma$ and $\lambda^{\prime}=\lambda_{\ell}^{\prime} \gamma$.

Let $T_{H}=\left\{\Xi_{H, n}\right\}$ denote the Voronoi tessellation induced by the points $X_{H, n}$ of the Cox process $X_{H}=\left\{X_{H, n}\right\}$, i.e.

$$
\Xi_{H, n}=\left\{x \in \mathbb{R}^{2}:\left|x-X_{H, n}\right| \leq\left|x-X_{H, m}\right| \text { for all } m \neq n\right\},
$$

where $|\cdot|$ denotes the Euclidean norm. The cells $\Xi_{H, n}$ of $T_{H}$ are considered to be the serving zones of HLCs. We can then construct the marked point process $X_{L, C}=\left\{\left(X_{L, n}, C_{n}\right)\right\}$, where the mark $C_{n}$ is the length of the shortest path from $X_{L, n}$ to $X_{H, j}$ along the edge set $T_{\gamma}^{(1)}$ of $T_{\gamma}$ provided that $X_{L, n} \in \Xi_{H, j}$. Thus, each LLC is connected to its nearest HLC in the Euclidean sense and not in the shortest path sense. However, for applications, this is a reasonable assumption since the planning of telecommunication networks is complicated and existing networks have evolved for long periods. Therefore, it is unrealistic to assume that serving zones are defined with respect to the shortest path distance and it is appropriate to use a simpler rule. Furthermore, analysis of real data has shown that the approach considered in the present paper is realistic (see [10]). Realizations of service zones and shortest paths are displayed in 

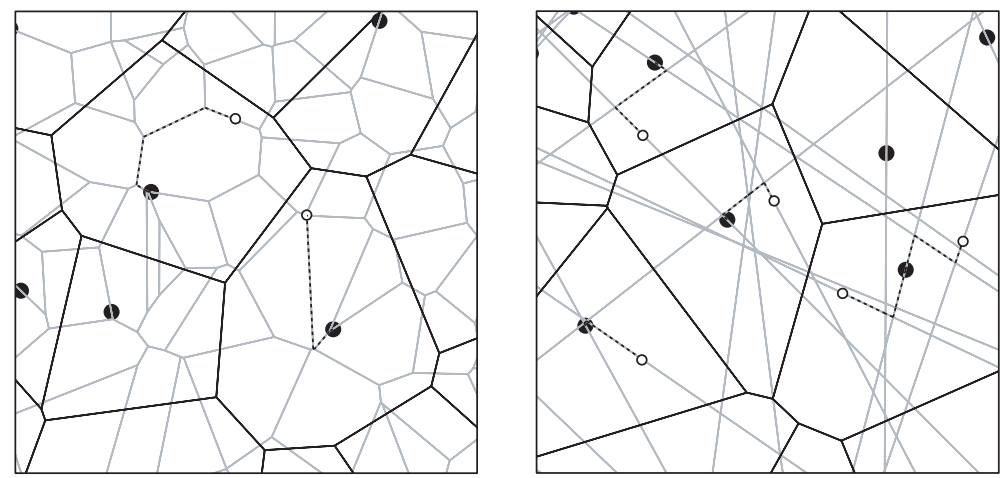

FIGURE 1: HLCs with serving zones (filled circles) and LLCs (open circles) with shortest paths (dashed lines) along the edge set (gray lines) of a PVT (left) and PLT (right).

Figure 1, where $T_{\gamma}$ is a Poisson-Voronoi tessellation (PVT) and a Poisson line tessellation (PLT), respectively.

The model characteristic we are mainly interested in is the distribution of the typical mark $C^{*}$ of $X_{L, C}$. Thus, we are interested in the Palm mark distribution $\mathrm{P}_{X_{L, C}}^{o}$ of $X_{L, C}$, i.e. the distribution of the typical shortest path length. Note that the realizations of $X_{L, C}$ can be constructed from the corresponding realizations of $X_{L}$ and $X_{H, S}$, where $X_{H, S}=\left\{\left(X_{H, n}, S_{H, n}^{o}\right)\right\}$ is a stationary marked point process with marks $S_{H, n}^{o}=\left(T_{\gamma}^{(1)} \cap \Xi_{H, n}\right)-X_{H, n}$. Thus, instead of $X_{L, C}$, we can consider the vector $Y=\left(X_{L}, X_{H, S}\right)$ and the Palm distribution $\mathrm{P}_{X_{L}}^{*}$ of $Y$ with respect to $X_{L}$, which has been introduced in $(2.1)$. Let $\left(X_{L}^{*}, \tilde{X}_{H, S}\right)$ be distributed according to $\mathrm{P}_{X_{L}}^{*}$, where we use the notation $\tilde{X}_{H, S}=\left\{\left(\tilde{X}_{H, n}, \tilde{S}_{H, n}^{o}\right)\right\}$ and

$$
\tilde{T}_{\gamma}^{(1)}=\bigcup_{n \geq 1}\left(\tilde{S}_{H, n}^{o}+\tilde{X}_{H, n}\right) .
$$

Note that $\tilde{X}_{H}=\left\{\tilde{X}_{H, n}\right\}$ is a (nonstationary) Cox process on $\tilde{T}_{\gamma}^{(1)}$ with linear intensity $\lambda_{\ell}$. Let $\tilde{X}_{H, 0}$ denote the closest point (in the Euclidean sense) of $\left\{\tilde{X}_{H, n}\right\}$ to the origin $o$. Then, the typical shortest path length $C^{*}$ can be given by $C^{*}=c\left(\tilde{X}_{H, 0}\right)$, where $c\left(\tilde{X}_{H, 0}\right)$ denotes the length of the shortest path from $o$ to $\tilde{X}_{H, 0}$ along $\tilde{T}_{\gamma}^{(1)}$. In the following we assume that the joint distribution of $C^{*}, \tilde{X}_{H}$, and $\tilde{T}_{\gamma}$ is given by $\mathrm{P}_{X_{L}}^{*}$.

\section{Limit theorems for the typical shortest path length}

We investigate the asymptotic behavior of the distribution of $C^{*}$ for two different cases: $\gamma / \lambda_{\ell} \rightarrow 0$ with $\lambda_{\ell}$ fixed and $\gamma / \lambda_{\ell} \rightarrow \infty$ with $\gamma \lambda_{\ell}$ fixed, i.e. unboundedly sparse edge sets and unboundedly dense edge sets, respectively. For $\gamma / \lambda_{\ell} \rightarrow 0$, we show in Theorem 3.1 that the distribution of $C^{*}$ converges weakly to an exponential distribution, where no specific assumption on the underlying stationary tessellation $T$ is needed. Furthermore, for $\gamma / \lambda_{\ell} \rightarrow \infty$ and stationary, isotropic, and mixing $T$, we show in Theorem 3.2 that $C^{*}$ converges weakly to a Weibull distribution.

\subsection{Scaling invariance property}

Recall that the stochastic network model introduced in Section 2 and, in particular, the distribution of $C^{*}$ is fully specified by $T, \gamma, \lambda_{\ell}$, and $\lambda_{\ell}^{\prime}$. Moreover, it can be shown (see, 

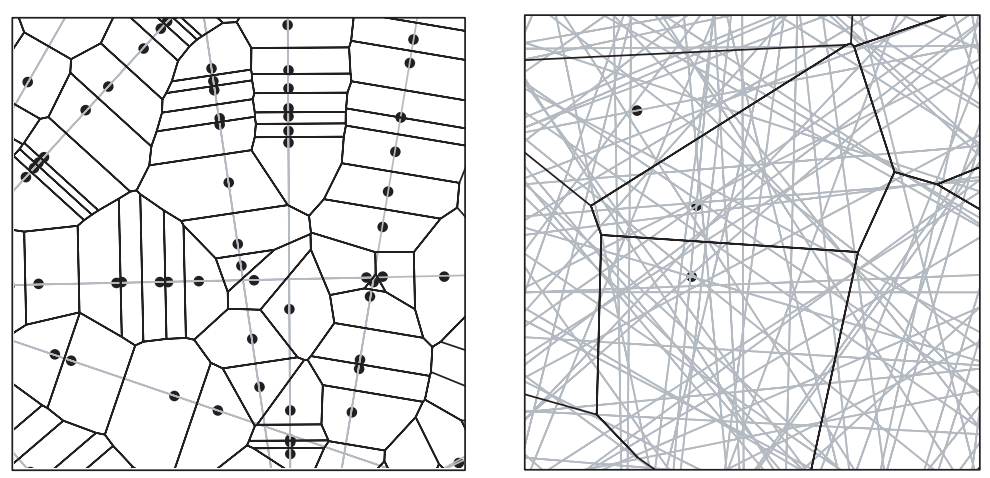

FIGURE 2: Realizations of $X_{H, S}=\left\{\left(X_{H, n}, S_{H, n}^{o}\right)\right\}$ for $\kappa=0.5$ (left $)$ and $\kappa=1000$ (right).

e.g. [14] and [33]) that the distribution of $C^{*}$ does not depend on $\lambda_{\ell}^{\prime}$. Therefore, we only regard the parameters $\gamma$ and $\lambda_{\ell}$ in the following. Sometimes we use the notation $C^{*}=C^{*}\left(\gamma, \lambda_{\ell}\right)$ to emphasize that the distribution of $C^{*}$ depends on $\gamma$ and $\lambda_{\ell}$.

Furthermore, a scaling invariance property holds for this model. If the value of the quotient $\kappa=\gamma / \lambda_{\ell}$ is constant then the structure of $X_{H, S}$ is fixed, but on different scales for different parameter vectors $\left(\gamma, \lambda_{\ell}\right)=\left(\kappa \lambda_{\ell}, \lambda_{\ell}\right)$. We are interested in the limiting behavior of $C^{*}$ for $\kappa \rightarrow 0$ with $\lambda_{\ell}$ fixed and for $\kappa \rightarrow \infty$ with $\lambda=\lambda_{\ell} \gamma$ fixed. In Figure 2 realizations of $X_{H, S}$ are shown for extremely small and large values of $\kappa$, where the realization of $T$ is sampled from a PLT. We can see that, for small $\kappa$, the segment systems within the serving zones mainly consist of one single segment only, whereas, for large $\kappa$, the networks inside the serving zones become rather dense.

\subsection{Asymptotic exponential distribution for $\kappa \rightarrow 0$}

First we regard the case in which $\kappa=\gamma / \lambda_{\ell} \rightarrow 0$ with $\lambda_{\ell}$ fixed, i.e. $\gamma \rightarrow 0$.

Theorem 3.1. Let $T$ be an arbitrary stationary tessellation. Then, for any fixed $\lambda_{\ell}>0$, it holds that

$$
C^{*}\left(\gamma, \lambda_{\ell}\right) \stackrel{\mathrm{D}}{\rightarrow} Z \quad \text { as } \gamma \rightarrow 0
$$

where $\stackrel{\mathrm{D}}{\rightarrow}$ ' denotes convergence in distribution and $Z \sim \operatorname{Exp}\left(2 \lambda_{\ell}\right)$, i.e. the random variable $Z$ is exponentially distributed with expectation $\left(2 \lambda_{\ell}\right)^{-1}$.

Proof. It can be shown that $C^{*}$ converges in distribution to the distance from $o$ to the nearest point of a stationary Poisson process of intensity $\lambda_{\ell}$ in $\mathbb{R}$ which is $\operatorname{Exp}\left(2 \lambda_{\ell}\right)$-distributed. For details, see [32].

Note that the case $\kappa \rightarrow 0$ with $\gamma$ fixed and $\lambda_{\ell} \rightarrow \infty$ can be treated as follows. Owing to the scaling invariance property mentioned in Section 3.1, we find, for $Z \sim \operatorname{Exp}(2)$, that

$$
\lambda_{\ell} C^{*}\left(\gamma, \lambda_{\ell}\right) \stackrel{\mathrm{D}}{=} C^{*}\left(\frac{\gamma}{\lambda_{\ell}}, 1\right) \stackrel{\mathrm{D}}{\rightarrow} Z \quad \text { as } \lambda_{\ell} \rightarrow \infty
$$

where ' $=$, denotes equality in distribution. 


\subsection{Asymptotic Weibull distribution for $\kappa \rightarrow \infty$}

In this section we assume that $T$ is a stationary and isotropic random tessellation which is mixing. Furthermore, we assume that

$$
\mathrm{E} v_{1}^{2}\left(\partial \Xi^{*}\right)<\infty
$$

where $v_{1}\left(\partial \Xi^{*}\right)$ denotes the circumference of the typical cell $\Xi^{*}$ of $T$. We investigate the asymptotic behavior of the distribution of $C^{*}=C^{*}\left(\gamma, \lambda_{\ell}\right)$ for $\kappa \rightarrow \infty$, where $\gamma \rightarrow \infty$ and $\lambda_{\ell} \rightarrow 0$ such that $\lambda_{\ell} \gamma=\lambda$ is fixed. In particular, we show that $C^{*}$ converges in distribution to $\xi Z$, where $\xi \geq 1$ is a certain constant which is multiplied by the Euclidean distance $Z$ from $o$ to the nearest point of a stationary Poisson process of intensity $\lambda$. Then, it is easy to see that $Z$ as well as $\xi Z$ have Weibull distributions.

Theorem 3.2. Let $Z \sim \operatorname{Wei}(\lambda \pi, 2)$ for some $\lambda>0$. Then there exists a constant $\xi \geq 1$ such that

$$
C^{*}\left(\gamma, \lambda_{\ell}\right) \stackrel{\mathrm{D}}{\rightarrow} \xi Z \quad \text { as } \kappa \rightarrow \infty
$$

provided that $\gamma \rightarrow \infty$ and $\lambda_{\ell} \rightarrow 0$ with $\lambda_{\ell} \gamma=\lambda$, where $\xi Z \sim \operatorname{Wei}\left(\lambda \pi / \xi^{2}, 2\right)$.

The proof of Theorem 3.2 is split into several steps. We first show in Lemma 4.2 that, under the Palm probability measure $\mathrm{P}_{X_{L}}^{*}$, the Euclidean distance $\left|\tilde{X}_{H, 0}\right|$ from $o$ to the nearest point $\tilde{X}_{H, 0}$ of the point process $\tilde{X}_{H}=\left\{\tilde{X}_{H, n}\right\}$ converges in distribution to the corresponding characteristic of a stationary Poisson process with intensity $\lambda$. Furthermore, in Lemma 4.4, we show that, for some constant $\xi \geq 1$, the difference between $\xi\left|\tilde{X}_{H, 0}\right|$ and the shortest path length $C^{*}=C^{*}\left(\gamma, \lambda_{\ell}\right)$ from the origin to $\tilde{X}_{H, 0}$ along $\tilde{T}_{\gamma}^{(1)}$ converges in probability to 0 . Then, combining the results of Lemmas 4.2 and 4.4, the assertion of Theorem 3.2 follows.

\section{Proof of Theorem 3.2}

\subsection{Euclidean distance from the typical LLC to its closest HLC}

Throughout this section, we assume that the underlying tessellation $T$ is ergodic. In order to prove that the Euclidean distance $\left|\tilde{X}_{H, 0}\right|$ from the typical LLC to its closest HLC is asymptotically Weibull distributed, we use classical results regarding the convergence in distribution of point processes; see, e.g. [8, Chapter 11] and [23, Chapters 7 and 10]. We first show that the Cox process $X_{H}$ converges in distribution to a homogeneous Poisson process $Y$ if $\kappa \rightarrow \infty$ provided that $\lambda_{\ell} \gamma=\lambda$ is constant, which we abbreviate as $X_{H} \Rightarrow Y$.

Lemma 4.1. If $\kappa \rightarrow \infty$, where $\lambda_{\ell} \gamma=\lambda$ for some constant $\lambda \in(0, \infty)$, then $X_{H} \Rightarrow Y$, where $Y$ is a stationary Poisson process with intensity $\lambda$.

Proof. For each $\gamma>1$, let $X_{H}=X_{H}(\gamma)$ denote the Cox process of HLCs with parameters $\gamma$ and $\lambda_{\ell}$, where $\lambda_{\ell}=\lambda / \gamma$ for some constant $\lambda \in(0, \infty)$. Note that the Cox process $X_{H}(\gamma)$ can be obtained from $X_{H}(1)$ by independent thinning with survival probability $c=1 / \gamma$ and by subsequent rescaling with scaling factor $\sqrt{1 / \gamma}$. Furthermore, the Cox process $X_{H}(1)$ is ergodic, since $T$ is ergodic. Thus, using, e.g. Theorem 7.3.1 of [23], we find that $X_{H}(\gamma) \Rightarrow Y$ as $\gamma \rightarrow \infty$.

Lemma 4.2. Let $Z \sim \operatorname{Wei}(\lambda \pi, 2)$ for some $\lambda>0$. Then $\left|\tilde{X}_{H, 0}\right| \stackrel{\mathrm{D}}{\rightarrow} Z$ as $\kappa \rightarrow \infty$ provided that $\gamma \rightarrow \infty$ and $\lambda_{\ell} \rightarrow 0$ such that $\lambda_{\ell} \gamma=\lambda$. 
Proof. Let $X_{H}^{*}(\gamma)$ be a point process in $\mathbb{R}^{2}$ whose distribution is equal to the Palm distribution of $X_{H}=X_{H}(\gamma)$. Furthermore, let $Y$ be a stationary Poisson process with intensity $\lambda$. Note that the distribution of $Y+\delta_{o}$ is then equal to the Palm distribution of $Y$; see, e.g. Proposition 13.1 of [8]. Thus, Proposition 10.3.6 of [23] yields

$$
X_{H}^{*}(\gamma) \Rightarrow Y+\delta_{o}
$$

as $\gamma \rightarrow \infty$ and $\lambda_{\ell} \rightarrow 0$, where $\lambda_{\ell} \gamma=\lambda$. Since $X_{L}$ and $X_{H}$ are Cox processes concentrated on $T_{\gamma}^{(1)}$ which are conditionally independent given $T_{\gamma}^{(1)}$, we find that $\tilde{X}_{H}+\delta_{0}$ and the Palm version $X_{H}^{*}$ of $X_{H}$ have the same distribution. This is an easy consequence of the representation formula for the Palm distribution of stationary Cox processes; see, e.g. Section 5.2 of [27]. In particular, this gives, for each $r>0$,

$$
\begin{aligned}
\lim _{\gamma \rightarrow \infty} \mathrm{P}\left(\left|\tilde{X}_{H, 0}\right|>r\right) & =\lim _{\gamma \rightarrow \infty} \mathrm{P}\left(\tilde{X}_{H}(B(o, r))=0\right) \\
& =\lim _{\gamma \rightarrow \infty} \mathrm{P}\left(\left(\tilde{X}_{H}+\delta_{o}\right)(B(o, r))=1\right) \\
& =\mathrm{P}\left(\left(Y+\delta_{0}\right)(B(o, r))=1\right) \\
& =\mathrm{P}(Y(B(o, r))=0),
\end{aligned}
$$

where we have used (4.1) in the penultimate equality. Thus, for each $r>0$,

$$
\lim _{\gamma \rightarrow \infty} \mathrm{P}\left(\left|\tilde{X}_{H, 0}\right|>r\right)=\mathrm{P}(Y(B(o, r))=0)=\exp \left(-\lambda \pi r^{2}\right),
$$

which means that $\left|\tilde{X}_{H, 0}\right| \stackrel{\mathrm{D}}{\rightarrow} Z \sim \operatorname{Wei}(\lambda \pi, 2)$.

\subsection{Shortest path length versus scaled Euclidean distance}

In this section we assume that $T$ is a stationary, isotropic, and mixing random tessellation. Furthermore, we assume that the integrability condition (3.1) is satisfied. Then, we can show that, for some constant $\xi \geq 1$, the difference between $\xi\left|\tilde{X}_{H, 0}\right|$ and the shortest path length $C^{*}=C^{*}\left(\gamma, \lambda_{\ell}\right)$ from $o$ to $\tilde{\tilde{X}}_{H, 0}$ along the edge set $\tilde{T}_{\gamma}^{(1)}$ converges in probability to 0 . In order to show this, we need the following auxiliary result.

Lemma 4.3. Let $\tilde{T}_{\gamma, \xi, \varepsilon}^{(1)}=\left\{u \in \tilde{T}_{\gamma}^{(1)}:|c(u)-\xi| u||<\varepsilon\right\}$, where $\xi \geq 1$ is some constant and $c(u)$ denotes the length of the shortest path from $u$ to the origin along the edges of $\tilde{T}_{\gamma}^{(1)}$. If $\gamma \rightarrow \infty$ and $\lambda_{\ell} \rightarrow 0$, where $\lambda_{\ell} \gamma=\lambda$ is fixed, then there exists $\xi \geq 1$ such that, for each $\varepsilon>0$ and $r>0$,

$$
\lim _{\gamma \rightarrow \infty} \operatorname{E} \exp \left(-\frac{\lambda}{\gamma} v_{1}\left(\tilde{T}_{\gamma}^{(1)} \backslash \tilde{T}_{\gamma, \xi, \varepsilon}^{(1)} \cap B(o, r)\right)\right)=1 .
$$

The proof of this lemma is postponed to Appendix A. Now, using Lemma 4.3, we are able to complete the proof of Theorem 3.2 by showing that the following is true.

Lemma 4.4. If $\gamma \rightarrow \infty$ and $\lambda_{\ell} \rightarrow 0$ such that $\lambda_{\ell} \gamma=\lambda$, then there exists a constant $\xi \geq 1$ with $C^{*}\left(\gamma, \lambda_{\ell}\right)-\xi\left|\tilde{X}_{H, 0}\right| \stackrel{\mathrm{P}}{\rightarrow} 0$, where $\stackrel{\text { ' }}{\rightarrow}$ ' denotes convergence in probability.

Proof. We have to show that there exists a constant $\xi \geq 1$ such that, for any $\varepsilon>0$ and $\delta>0$, we can choose $\gamma_{0}>0$ with $\mathrm{P}\left(\left|C^{*}-\xi\right| \tilde{X}_{H, 0}||>\varepsilon\right) \leq \delta$ for all $\gamma>\gamma_{0}$. Note that

$$
\begin{aligned}
\mathrm{P}\left(\left|C^{*}-\xi\right| \tilde{X}_{H, 0}||>\varepsilon\right)= & \mathrm{P}\left(\left|C^{*}-\xi\right| \tilde{X}_{H, 0}||>\varepsilon,\left|\tilde{X}_{H, 0}\right| \leq r\right) \\
& +\mathrm{P}\left(\left|C^{*}-\xi\right| \tilde{X}_{H, 0}||>\varepsilon,\left|\tilde{X}_{H, 0}\right|>r\right),
\end{aligned}
$$


where $r>0$ is an arbitrary fixed number. Since

$$
\mathrm{P}\left(\left|\tilde{X}_{H, 0}\right|>r\right) \rightarrow \mathrm{e}^{-\lambda \pi r^{2}} \quad \text { as } \gamma \rightarrow \infty
$$

(see Lemma 4.2), we can choose $r>0$ such that $\mathrm{P}\left(\left|\tilde{X}_{H, 0}\right|>r\right)<\delta / 2$ for all $\gamma>0$ sufficiently large. Thus, it is enough to show that there exists $\gamma_{0}>0$ such that $\mathrm{P}\left(\left|C^{*}-\xi\right| \tilde{X}_{H, 0}||>\varepsilon\right.$, $\left.\left|\tilde{X}_{H, 0}\right| \leq r\right) \leq \delta / 2$ for all $\gamma>\gamma_{0}$. Let $\tilde{N}=\tilde{X}_{H}(B(o, r))$ denote the number of points of $\tilde{X}_{H}$ in $B(o, r)$. Then we have

$$
\begin{aligned}
& \mathrm{P}\left(\left|C^{*}-\xi\right| \tilde{X}_{H, 0}||>\varepsilon,\left|\tilde{X}_{H, 0}\right| \leq r\right) \\
& \quad \leq \mathrm{E}\left(\sum_{k=1}^{\infty} \mathrm{P}\left(\tilde{N}=k \mid \tilde{T}_{\gamma}\right) \mathrm{P}\left(\max _{i=1, \ldots, k}\left(\left|c\left(Y_{i}\right)-\xi\right| Y_{i}||\right)>\varepsilon \mid \tilde{T}_{\gamma}, \tilde{N}=k\right)\right) \\
& \quad=\mathrm{E}\left(\sum_{k=1}^{\infty} \mathrm{P}\left(\tilde{N}=k \mid \tilde{T}_{\gamma}\right)\left(1-\mathrm{P}\left(\left|c\left(Y_{1}\right)-\xi\right| Y_{1}|| \leq \varepsilon \mid \tilde{T}_{\gamma}\right)^{k}\right)\right)
\end{aligned}
$$

where the points $Y_{1}, \ldots, Y_{k}$ are conditionally independent and identically distributed according to $v_{1}\left(\cdot \cap \tilde{T}_{\gamma}^{(1)} \cap B(o, r)\right) / \nu_{1}\left(\tilde{T}_{\gamma}^{(1)} \cap B(o, r)\right)$ for given $\tilde{T}_{\gamma}$ and $\tilde{N}=k$. In particular, for the conditional probability in the latter expression, we have

$$
\mathrm{P}\left(\left|c\left(Y_{1}\right)-\xi\right| Y_{1}|| \leq \varepsilon \mid \tilde{T}_{\gamma}\right)=\frac{\nu_{1}\left(\tilde{T}_{\gamma, \xi, \varepsilon}^{(1)} \cap B(o, r)\right)}{v_{1}\left(\tilde{T}_{\gamma}^{(1)} \cap B(o, r)\right)} .
$$

Using the fact that $\tilde{N} \sim \operatorname{Po}(\tilde{\lambda})$ with $\tilde{\lambda}=\lambda_{\ell} v_{1}\left(\tilde{T}_{\gamma}^{(1)} \cap B(o, r)\right)$ given $\tilde{T}_{\gamma}$, we obtain

$$
\begin{aligned}
\sum_{k=1}^{\infty} \mathrm{P}\left(\tilde{N}=k \mid \tilde{T}_{\gamma}\right)\left(1-\mathrm{P}\left(\left|c\left(Y_{1}\right)-\xi\right| Y_{1}|| \leq \varepsilon \mid \tilde{T}_{\gamma}\right)^{k}\right) \\
=1-\sum_{k=0}^{\infty} \mathrm{e}^{-\tilde{\lambda}} \frac{\tilde{\lambda}^{k}}{k !}\left(\frac{\lambda_{\ell} v_{1}\left(\tilde{T}_{\gamma, \xi, \varepsilon}^{(1)} \cap B(o, r)\right)}{\tilde{\lambda}}\right)^{k} \\
=1-\exp \left(-\lambda_{\ell}\left(v_{1}\left(\tilde{T}_{\gamma}^{(1)} \cap B(o, r)\right)-v_{1}\left(\tilde{T}_{\gamma, \xi, \varepsilon}^{(1)} \cap B(o, r)\right)\right)\right) .
\end{aligned}
$$

Thus, we have

$\lim _{\gamma \rightarrow \infty} \mathrm{P}\left(\left|C^{*}-\xi\right| \tilde{X}_{H, 0}||>\varepsilon,\left|\tilde{X}_{H, 0}\right| \leq r\right) \leq 1-\lim _{\gamma \rightarrow \infty} \operatorname{E} \exp \left(-\frac{\lambda}{\gamma} \nu_{1}\left(\tilde{T}_{\gamma}^{(1)} \backslash \tilde{T}_{\gamma, \xi, \varepsilon}^{(1)} \cap B(o, r)\right)\right)$.

Hence, using Lemma 4.3, this gives $\lim _{\gamma \rightarrow \infty} \mathrm{P}\left(\left|C^{*}-\xi\right| \tilde{X}_{H, 0}||>\varepsilon,\left|\tilde{X}_{H, 0}\right| \leq r\right)=0$.

\section{Examples}

Recall that in Theorem 3.2 we assumed that the underlying tessellation $T$ is stationary, isotropic, mixing, and fulfills the integrability condition (3.1). The tessellation models considered in the literature focus mainly on PLT, PVT, Poisson-Delaunay tessellations (PDT), iterated tessellations constructed from these basic tessellations of Poisson type, and STIT tessellations; see, e.g. [1]-[5], [9]-[14], and [30]-[35]. Here, we assume that an iterated tessellation is either a $T_{I} / T_{I I}$-superposition or a $T_{I} / T_{I I}$-nesting of tessellations $T_{I}$ and $T_{I I}$ as defined in, e.g. [3], [22], and [35]. We show that for these important models Theorem 3.2 can be applied. 
Furthermore, if $T$ is a PLT or a $T_{I} / T_{I I}$-superposition/nesting with $T_{I}$ being a PLT, then we can even calculate the constant $\xi$ explicitly that appears in Theorem 3.2. On the other hand, if $T$ is a PDT, we obtain an upper bound for $\xi$.

Corollary 5.1. Let $Z \sim \operatorname{Wei}(\lambda \pi, 2)$, and let $T$ be a PDT, PVT, PLT, STIT, or an iterated tessellation $T=T_{I} / T_{I I}$ such that $T_{I}$ and $T_{I I}$ fulfill (3.1), where $T$ is either

1. a superposition of two mixing tessellations $T_{I}$ and $T_{I I}$, or

2. a nesting of a mixing tessellation $T_{I}$ and a stationary tessellation $T_{I I}$.

Then $C^{*} \stackrel{\mathrm{D}}{\rightarrow} \xi Z$ for some constant $\xi \geq 1$ provided that $\gamma \rightarrow \infty$ and $\lambda_{\ell} \rightarrow 0$ such that $\lambda_{\ell} \gamma=\lambda$. Furthermore, if $T$ is a PLT or a $T_{I} / T_{I I}$-superposition/nesting, where $T_{I}$ is a PLT, then $\xi=1$. If $T$ is a PDT then $\xi \leq 4 / \pi \approx 1.27$.

Proof. The first part of the assertion follows from Theorem 3.2 since all considered tessellations are mixing (see [20], [26, Chapter 10.5], and [32]) and fulfill the integrability condition (3.1) (see [32]).

Now we consider the case in which $T$ is a PLT with intensity 1 . Then, the edge set $\tilde{T}_{\gamma}^{(1)}$ of $\tilde{T}_{\gamma}$ is generated by a random sequence of lines $L_{0}, L_{1}, \ldots$, where $L_{1}, L_{2}, \ldots$ form the edge set $T_{\gamma}^{(\mathrm{I})}$ of the (stationary and isotropic) PLT $T_{\gamma}$ and $L_{0}$ is an isotropic line through the origin $o$, which is independent of $T_{\gamma}$. Thus, we have

$$
\frac{1}{\gamma} v_{1}\left(\tilde{T}_{\gamma}^{(1)} \backslash \tilde{T}_{\gamma, \xi, \varepsilon}^{(1)} \cap B(o, r)\right) \leq \frac{1}{\gamma} v_{1}\left(T_{\gamma}^{(1)} \cap B(o, r)\right)+\frac{2 r}{\gamma} .
$$

Using Theorem 2.12.4 of [6], it can be shown that the family of random variables $\left\{X_{\gamma, \xi}, \gamma>0\right\}$ with

$$
X_{\gamma, \xi}=\frac{1}{\gamma} v_{1}\left(\tilde{T}_{\gamma}^{(1)} \backslash \tilde{T}_{\gamma, \xi, \varepsilon}^{(1)} \cap B(o, r)\right)
$$

is uniformly integrable since

$$
\frac{1}{\gamma} v_{1}\left(T_{\gamma}^{(1)} \cap B(o, r)\right)=\pi r^{2} \frac{\nu_{1}\left(T^{(1)} \cap B(o, r \gamma)\right)}{\nu_{2}(B(o, r \gamma))}
$$

converges to $r^{2} \pi$ in $L^{1}$ due to the fact that the PLT $T$ is mixing and, therefore, ergodic (see [8, Theorem 12.2.IV]). Furthermore, in Lemma 4.3 we showed that there exists a $\xi \geq 1$ such that the Laplace transform of $X_{\gamma, \xi}$ converges to 1 , which implies that $X_{\gamma, \xi} \stackrel{\mathrm{P}}{\rightarrow} 0$ (see [18, Theorem 5.3]). Thus, applying Theorem 2.12.4 of [6] again, we obtain

$$
\lim _{\gamma \rightarrow \infty} \mathrm{E} X_{\gamma, \xi}=0
$$

However, if $T_{\gamma}^{(1)}$ gets denser, there are lines which intersect $L_{0}$ close to $o$. Thus, all points on these lines have approximately the direct connections as shortest paths. This can be used to show that $\xi=1$. Suppose that $\xi>1$, and let $r>2>\varepsilon>0$ with $\xi>1+\varepsilon$. If the line $L_{i}$ intersects the segment $L_{0, \varepsilon}$, where $L_{0, \varepsilon}=L_{0} \cap B(o, \varepsilon / 2)$, then, for each $y \in L_{i}$, it holds that $0 \leq c(y)-|y| \leq \varepsilon$ since the path from $y$ to $o$ via the intersection point $L_{i} \cap L_{0, \varepsilon}$ is not longer than $|y|+\varepsilon$. Thus, if $|y|>2$,

$$
|c(y)-\xi| y||=|c(y)-| y|-(\xi-1)| y|| \geq \varepsilon(|y|-1) \geq \varepsilon,
$$


which means that $y \in \tilde{T}_{\gamma}^{(1)} \backslash \tilde{T}_{\gamma, \xi, \varepsilon}^{(1)}$. Furthermore, if $L_{i} \cap L_{0, \varepsilon} \neq \varnothing$, it is easy to see that $v_{1}\left(L_{i} \cap B(o, r) \backslash B(o, 2)\right) \geq a$ for some constant $a>0$. These two observations lead to

$$
\begin{aligned}
X_{\gamma, \xi} & =\frac{1}{\gamma} v_{1}\left(\tilde{T}_{\gamma}^{(1)} \backslash \tilde{T}_{\gamma, \xi, \varepsilon}^{(1)} \cap B(o, r)\right) \\
& \geq \frac{1}{\gamma} v_{1}\left(\bigcup_{\left\{i: L_{i} \cap L_{0, \varepsilon} \neq \varnothing\right\}}\left\{L_{i} \cap B(o, r) \backslash B(o, 2)\right\}\right) \\
& \geq \frac{a}{\gamma} \#\left\{L_{i}: L_{i} \cap L_{0, \varepsilon} \neq \varnothing\right\},
\end{aligned}
$$

and, since $\#\left\{L_{i}: L_{i} \cap L_{0, \varepsilon} \neq \varnothing\right\} \sim \operatorname{Po}(2 \varepsilon \gamma / \pi)$,

$$
\liminf _{\gamma \rightarrow \infty} \mathrm{E} X_{\gamma, \xi} \geq \lim _{\gamma \rightarrow \infty} \frac{a}{\gamma} \mathrm{E} \#\left\{L_{i}: L_{i} \cap L_{0, \varepsilon} \neq \varnothing\right\}=\frac{2 \varepsilon a}{\pi}>0,
$$

which is in contradiction to (5.1). Thus, $\xi=1$ holds. If $T$ is a superposition/nesting such that $T_{I}$ is a PLT, then basically the same arguments yield $\xi=1$. Finally, let $T$ be a PDT and let $N(y)$ denote that node of $T$ which is closest to $y \in \mathbb{R}^{2}$. It has been shown in [4] that, for any $t>0$ and $y \in \partial B(o, 1)$, there is a path $P(t y)$ from $N(o)$ to $N(t y)$ on $T^{(1)}$ with length $c(P(t y))$ such that, almost surely, $c(P(t y)) / t \rightarrow 4 / \pi$ as $t \rightarrow \infty$, which yields $\xi \leq 4 / \pi$.

\section{Conclusion and outlook}

We considered the typical shortest path length $C^{*}$ of stochastic network models with two hierarchy levels, where the locations of network components are modeled by Cox processes on the edges of random tessellations. It was shown that the distribution of $C^{*}$ converges to known limit distributions for extreme cases of the model parameters, i.e. if a certain scaling factor $\kappa$ tends to 0 or $\infty$.

The results of the present paper have applications in the analysis of telecommunication access networks since the distribution of $C^{*}$ is closely related to cost and risk analysis of such networks (see [10]). Using the fitting techniques introduced in [13], an optimal tessellation model can be chosen for a given set of road data. Moreover, the scaling factor $\kappa$ can be estimated. Then, on the one hand, for small values of $\kappa$, the limit distribution of $C^{*}$ is directly available and it does not depend on the type of optimal tessellation model. On the other hand, for large values of $\kappa$, the limit distribution of $C^{*}$ and an upper bound for this distribution is directly available if the optimal model is PLT or PLT-superposition/nesting and PDT, respectively.

In order to get an idea of how small or large the scaling factor $\kappa$ should be (to replace the distribution of $C^{*}$ by the corresponding limit distribution) and how to calculate the constant $\xi$ appearing in the limit distribution for $C^{*}$ as $\kappa \rightarrow \infty$, the density of $C^{*}$ can be estimated by Monte Carlo simulation of the typical serving zone (see [33]). This can be done for PVT, PLT, and PDT as well as for superpositions and nestings built from these basic tessellation models, using simulation algorithms of the typical serving zone introduced in [9], [12], [31], and [34]. In Figures 3 and 4 estimated densities for different values of $\kappa$ are shown together with the corresponding limit distributions if the tessellation model chosen for the underlying road system is a PLT and PVT, respectively. As can be seen in Figure 4(b), the density of the Wei $\left(\lambda \pi / 1.145^{2}, 2\right)$-distribution approximates the density of $C^{*}$ very well for $T$ being a PVT and $\kappa \geq 1000$. This suggests that in this case the constant $\xi$ appearing in Theorem 3.2 is approximately 1.145 . 

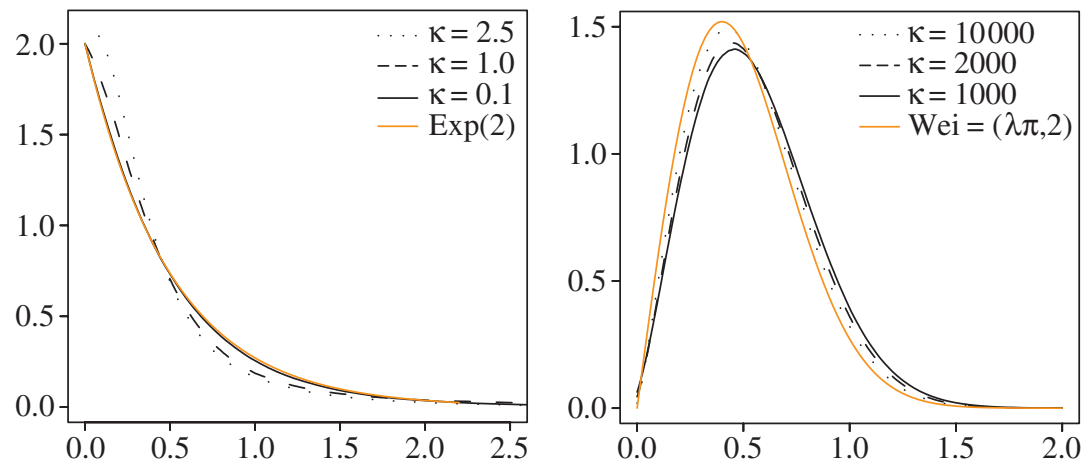

FIgURE 3: Densities of $C^{*}$ if $T$ is a PLT (together with the corresponding limit distributions).
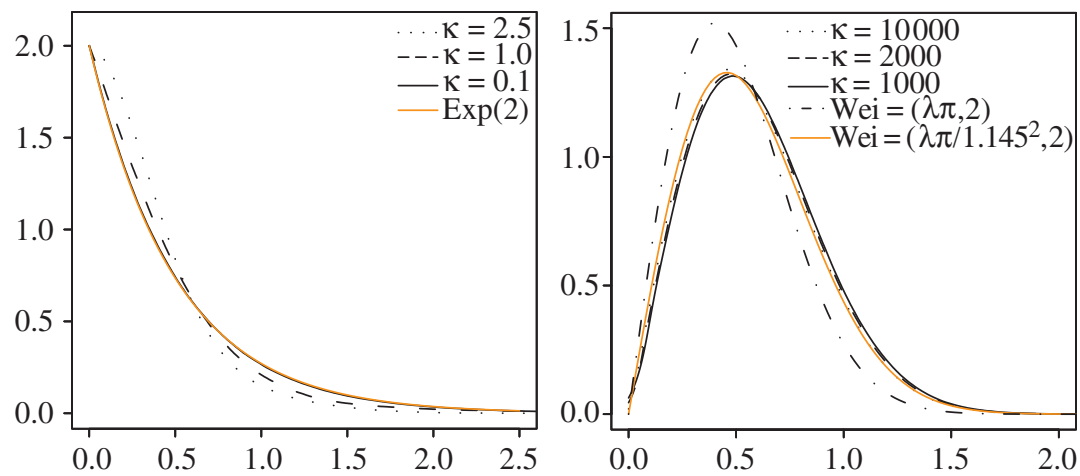

Figure 4: Densities of $C^{*}$ if $T$ is a PVT (together with the corresponding limit distributions).

Furthermore, the limiting distributions derived in the present paper can be used to choose parametric densities which can be fitted to the estimated density of $C^{*}$ for a large range of $\kappa$. Parametric families which include both exponential and Weibull distributions turned out to be good choices; see [10]. In Figure 5 estimated densities for different values of $\kappa$ are shown together with fitted truncated Weibull distributions. These truncated Weibull distributions have two parameters and there is quite a good fit for both tessellation models considered in Figure 5 and a large range of values of $\kappa$.

Note that the setting of Theorem 3.2 can be generalized in different ways. For example, the statement of this theorem remains valid if instead of $C^{*}$ the typical subscriber line length $S^{*}$ is considered, where $S^{*}$ is the shortest path length from the origin to the nearest point $X_{H, 0}$ of $X_{H}$, which is defined as the sum of the distance from the origin to the nearest point of the edge set $T^{(1)}$ and the shortest path length on $T^{(1)}$ from this point to $X_{H, 0}$ (see [14]). In this case the auxiliary results corresponding to Lemmas 4.3 and 4.4 can basically be proved in the same way.

Furthermore, in the proof of Theorem 3.2 it is not necessary to assume that $T$ is a random tessellation, but it is possible to consider a stationary and isotropic segment process in $\mathbb{R}^{2}$ which is mixing and such that there is only one single cluster with probability 1 . In particular, Theorem 3.2 can be extended to random geometric graphs. 

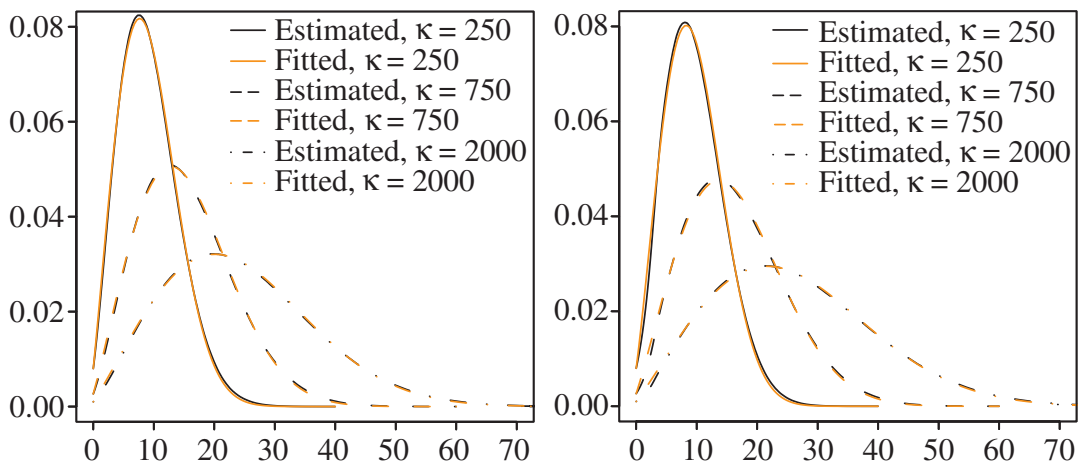

Figure 5: Densities of $C^{*}$ (with fitted parametric densities) for a PLT (left) and PVT (right).

Another kind of extension can be obtained by relaxing the assumption that $X_{L, n}$ is connected to the nearest point of $X_{H}$, i.e. $T_{H}$ is a Voronoi tessellation. For instance, $X_{L, n}$ can be connected to its $k$ th nearest neighbor of $X_{H}$ for any $k \geq 1$. Then, in Theorem 3.2 we only have to replace $Z$ by the distance from the origin to the $k$ th nearest point of a Poisson process which is distributed according to a generalized Gamma distribution (see [15] and [36]). Further possible extensions include that $T_{H}$ is a certain Cox-Laguerre tessellation (see [21]) or an aggregated tessellation (see [3] and [28]).

\section{Appendix A. Proof of Lemma 4.3}

Obviously,

$$
\limsup _{\gamma \rightarrow \infty} E \exp \left(-\frac{\lambda}{\gamma} v_{1}\left(\tilde{T}_{\gamma}^{(1)} \backslash \tilde{T}_{\gamma, \xi, \varepsilon}^{(1)} \cap B(o, r)\right)\right) \leq 1 .
$$

Thus, it is sufficient to show that

$$
\liminf _{\gamma \rightarrow \infty} \operatorname{E} \exp \left(-\frac{\lambda}{\gamma} v_{1}\left(\tilde{T}_{\gamma}^{(1)} \backslash \tilde{T}_{\gamma, \xi, \varepsilon}^{(1)} \cap B(o, r)\right)\right) \geq 1 .
$$

Proof of (A.1). First recall that we can identify $\tilde{T}_{\gamma}^{(1)}$ with the Palm version $\Lambda_{T_{\gamma}^{(1)}}^{*}$ of the stationary random measure $\Lambda_{T_{\gamma}^{(1)}}$ given by $\Lambda_{T_{\gamma}^{(1)}}(B)=v_{1}\left(B \cap T_{\gamma}^{(1)}\right)$ for $B \in \mathcal{B}^{2}$ since $\Lambda_{T_{\gamma}^{(1)}}$ is the random driving measure of the Cox process $X_{L}$; see [27, p. 156]. Then, using the abbreviation

$$
h\left(\tau^{(1)}\right)=\exp \left(-\frac{\lambda}{\gamma} v_{1}\left(\tau^{(1)} \backslash \tau_{\xi, \varepsilon}^{(1)} \cap B(o, r)\right)\right),
$$

where $\tau_{\xi, \varepsilon}^{(1)}=\left\{u \in \tau^{(1)}:|c(u)-\xi| u||<\varepsilon\right\}$ and $c(u)$ denotes the length of the shortest path from $u$ to $o$ along the edge set $\tau^{(1)}$ of a tessellation $\tau$ with $o \in \tau^{(1)}$, we obtain, from the Campbell theorem for stationary random measures (see [8, Proposition 13.2.V]),

$$
\begin{aligned}
\mathrm{E} h\left(\tilde{T}_{\gamma}^{(1)}\right) & =\frac{1}{\gamma \nu_{2}(B(o, 1 / \gamma))} \mathrm{E}\left(\int_{T_{\gamma}^{(1)} \cap B(o, 1 / \gamma)} h\left(T_{\gamma}^{(1)}-x\right) \nu_{1}(\mathrm{~d} x)\right) \\
& =\frac{1}{\pi} \mathrm{E}\left(\int_{T^{(1)} \cap B(o, 1)} h\left(T_{\gamma}^{(1)}-\frac{z}{\gamma}\right) v_{1}(\mathrm{~d} z)\right),
\end{aligned}
$$


where we have used the substitution $z=\gamma x$ in the last expression, bearing in mind that $(1 / \gamma) T^{(1)}=T_{\gamma}^{(1)}$. Furthermore, we set $T_{\gamma, \varepsilon, z}^{(1)}=\left\{y \in T_{\gamma}^{(1)}:|c(y, z / \gamma)-\xi| y-z / \gamma||<\varepsilon\right\}$, where $c(y, z / \gamma)$ denotes the length of the shortest path from $y$ to $z / \gamma$ along the edges of the considered graph. Then, for each $\gamma \geq 1$, we obtain

$$
\begin{aligned}
\mathrm{E} h\left(\tilde{T}_{\gamma}^{(1)}\right)= & \frac{1}{\pi} \mathrm{E}\left(\int_{T^{(1)} \cap B(o, 1)} \exp \left(-\frac{\lambda}{\gamma} v_{1}\left(T_{\gamma}^{(1)} \backslash T_{\gamma, \varepsilon, z}^{(1)} \cap B\left(\frac{z}{\gamma}, r\right)\right)\right) v_{1}(\mathrm{~d} z)\right) \\
\geq \frac{1}{\pi} \mathrm{E}( & v_{1}\left(T^{(1)} \cap B(o, 1)\right) \\
& \left.\quad \times \inf _{z \in T^{(1)} \cap B(o, 1)} \exp \left(-\frac{\lambda}{\gamma} v_{1}\left(T_{\gamma}^{(1)} \backslash T_{\gamma, \varepsilon, z}^{(1)} \cap B\left(\frac{z}{\gamma}, r\right)\right)\right)\right) \\
\geq \frac{1}{\pi} \mathrm{E}( & v_{1}\left(T^{(1)} \cap B(o, 1)\right) \\
& \left.\quad \times \exp \left(-\sup _{z \in T^{(1)} \cap B(o, 1)} \frac{\lambda}{\gamma} v_{1}\left(T_{\gamma}^{(1)} \backslash T_{\gamma, \varepsilon, z}^{(1)} \cap B(o, r+1)\right)\right)\right) .
\end{aligned}
$$

Now, in order to prove (A.1), it is sufficient to show that

$$
X_{\gamma, \xi} \stackrel{L^{1}}{\rightarrow} 0 \text { for } \gamma \rightarrow \infty
$$

where $X_{\gamma, \xi}=\sup _{z \in T^{(1)} \cap B(o, 1)}(1 / \gamma) \nu_{1}\left(T_{\gamma}^{(1)} \backslash T_{\gamma, \varepsilon, z}^{(1)} \cap B(o, r+1)\right)$. To see this, note that (A.2) implies that the random variable $Y_{\gamma, \xi}=\exp \left(-\lambda X_{\gamma, \xi}\right) \nu_{1}\left(T^{(1)} \cap B(o, 1)\right)$ converges in probability to $v_{1}\left(T^{(1)} \cap B(o, 1)\right)$. Moreover, $Y_{\gamma, \xi} \leq v_{1}\left(T^{(1)} \cap B(o, 1)\right)$ for all $\gamma \geq 1$ and $\mathrm{E} v_{1}\left(T^{(1)} \cap\right.$ $B(o, 1))=\pi<\infty$. Thus, $\left\{Y_{\gamma, \xi}, \gamma \geq 1\right\}$ is uniformly integrable and Theorem 2.12.4 of [6] yields $\lim _{\gamma \rightarrow \infty} 1 / \pi \mathrm{E} Y_{\gamma, \xi}=1$, which shows that (A.1) is true.

Proof of (A.2). Since $X_{\gamma, \xi} \geq 0$, it suffices to show that $\mathrm{E} X_{\gamma, \xi} \rightarrow 0$. Furthermore, note that, with probability 1 , the segments of the segment system $T_{\gamma}^{(1)} \cap B(o, r+1)$ fulfill the conditions of the generalized Blaschke-Petkantschin formula (see [17, Proposition 5.4]). Thus, using the notation $L_{\Phi}^{+}$for the half line with direction $\Phi$ through $o$, we obtain

$$
\begin{aligned}
& \mathrm{E} X_{\gamma, \xi}=\mathrm{E}\left(\frac{1}{\gamma} \sup _{z \in T^{(1)} \cap B(o, 1)} \int_{0}^{2 \pi} \sum_{\left\{X_{i} \in T_{\gamma}^{(1)} \cap L_{\Phi}^{+}:\left|X_{i}\right| \leq r+1\right\}} \frac{\left|X_{i}\right|}{\sin \alpha_{i}} \mathbf{1}_{[\varepsilon, \infty)}\right. \\
& \left.\times\left(\left|c\left(X_{i}, \frac{z}{\gamma}\right)-\xi\right| X_{i}-\frac{z}{\gamma}||\right) \mathrm{d} \Phi\right) \\
& \leq \frac{r+1}{\gamma} \\
& \times \mathrm{E}\left(\int_{0}^{2 \pi} \sup _{z \in T^{(1)} \cap B(o, 1)} \sum_{\left\{X_{i} \in T_{\gamma}^{(1)} \cap L_{\Phi}^{+}:\left|X_{i}\right| \leq r+1\right\}} \frac{1}{\sin \alpha_{i}} \mathbf{1}_{[\varepsilon, \infty)}\right. \\
& \left.\times\left(\left|c\left(X_{i}, \frac{z}{\gamma}\right)-\xi\right| X_{i}-\frac{z}{\gamma}||\right) \mathrm{d} \Phi\right) \\
& =2 \pi(r+1) \mathrm{E} g_{\gamma}\left(T^{(1)}\right),
\end{aligned}
$$


where in the last line we used Fubini's theorem, the isotropy of $T$, and the abbreviation

$$
g_{\gamma}\left(T^{(1)}\right)=\frac{1}{\gamma} \sup _{z \in T^{(1)} \cap B(o, 1)} \sum_{\left\{X_{i} \in T_{\gamma}^{(1)} \cap L^{+}:\left|X_{i}\right| \leq r+1\right\}} \frac{1}{\sin \alpha_{i}} \mathbf{1}_{[\varepsilon, \infty)}\left(\left|c\left(X_{i}, \frac{z}{\gamma}\right)-\xi\right| X_{i}-\frac{z}{\gamma}||\right)
$$

with $L^{+}=L_{0}^{+}$. Since the point process $T^{(1)} \cap \mathbb{R}$ is stationary with intensity $2 / \pi$ (see [26, Theorem 4.5.3]), where we identify $\mathbb{R}$ with the $x$-axis, we can apply the inversion formula for Palm distributions of stationary point processes on $\mathbb{R}$; see Proposition 11.3(iii) of [18]. Thus, if $T^{(1) *}$ denotes the Palm version of $T^{(1)}$ with respect to the point process $T^{(1)} \cap \mathbb{R}$, we obtain

$$
\mathrm{E} g_{\gamma}\left(T^{(1)}\right)=\frac{2}{\pi} \mathrm{E}\left(\int_{0}^{\infty} \mathbf{1}_{\left[0, X_{1}^{*}\right]}(x) g_{\gamma}\left(T^{(1) *}-x\right) \mathrm{d} x\right),
$$

where the points of $\left\{X_{i}^{*}\right\}=T^{(1) *} \cap \mathbb{R}$ are numbered in ascending order such that $\cdots<X_{-1}^{*}<$ $X_{0}^{*}=0<X_{1}^{*}<X_{2}^{*}<\cdots$. Hence, in order to prove (A.2), it suffices to show that

$$
\lim _{\gamma \rightarrow \infty} \mathrm{E}\left(\int_{0}^{\infty} \mathbf{1}_{\left[0, X_{1}^{*}\right]}(x) g_{\gamma}\left(T^{(1) *}-x\right) \mathrm{d} x\right)=0 .
$$

The proof of (A.3) is subdivided into two main steps. First, we show that

$$
\lim _{\gamma \rightarrow \infty} \tilde{g}_{\gamma}\left(x, T^{(1) *}\right)=0
$$

almost everywhere with respect to the product measure $v_{1} \otimes \mathrm{P}^{*}$, where we have used the abbreviating notation $\tilde{g}_{\gamma}\left(x, T^{(1) *}\right)=\mathbf{1}_{\left[0, X_{1}^{*}\right]}(x) g_{\gamma}\left(T^{(1) *}-x\right)$ and $\mathrm{P}^{*}$ denotes the distribution of $T^{(1) *}$. Then, we show that $\left\{\tilde{g}_{\gamma}, \gamma>0\right\}$ is uniformly $\left(v_{1} \otimes \mathrm{P}^{*}\right)$-integrable. By means of Theorem 2.12.4 of [6], this implies that (A.3) holds.

Proof of (A.4). Note that, for each $x \in\left[0, X_{1}^{*}\right]$, we obtain

$$
\begin{aligned}
& g_{\gamma}\left(T^{(1) *}-x\right) \\
& \leq \frac{1}{\gamma} \sup _{z \in\left(T^{(1) *}-x\right) \cap B(o, 1)} \sum_{\left\{X_{i} \in\left(T_{\gamma}^{(1) *}-x / \gamma\right) \cap L^{+}:\left|X_{i}\right| \leq r+1\right\}} \frac{1}{\sin \alpha_{i}} \mathbf{1}_{[\varepsilon, \infty)} \\
& \times\left(\left|c\left(X_{i}, \frac{z}{\gamma}\right)-\xi\right| X_{i}-\frac{z}{\gamma}||\right) \\
& \leq \frac{1}{\gamma} \sum_{\left\{X_{i}^{*} \in T^{(1) *} \cap\left(L^{+}+x\right): X_{i}^{*} \in B(x,(r+1) \gamma)\right\}} \frac{1}{\sin \alpha_{i}} \sup _{z \in T^{(1) *} \cap B(x, 1)} \mathbf{1}_{[\varepsilon, \infty)} \\
& \times\left(\frac{1}{\gamma}\left|c\left(X_{i}^{*}, z\right)-\xi\right| X_{i}^{*}-z||\right)
\end{aligned}
$$

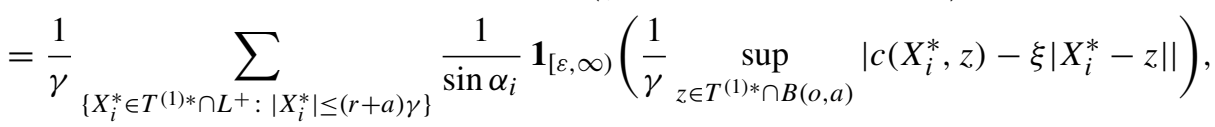

where $a=1+X_{1}^{*}$. Furthermore, we have

$$
\begin{aligned}
\frac{1}{\gamma} \sup _{z \in T^{(1) * \cap B(o, a)}}\left|c\left(X_{i}^{*}, z\right)-\xi\right| X_{i}^{*}-z|| \leq & \frac{1}{\gamma}\left(c\left(o, X_{i}^{*}\right)-\xi\left|X_{i}^{*}\right|\right) \\
& +\frac{1}{\gamma}\left(\sup _{z \in T^{(1) * \cap B(o, a)}} c(z, o)+\xi a\right) .
\end{aligned}
$$


Clearly, the second term of this upper bound tends to zero $\mathrm{P}^{*}$-almost surely as $\gamma \rightarrow \infty$. Thus, in order to show that (A.4) holds, it suffices to prove that, $\mathrm{P}^{*}$-almost surely,

$$
\frac{1}{\gamma}\left(c\left(o, X_{i}^{*}\right)-\xi X_{i}^{*}\right) \in\left(-\frac{\varepsilon}{2}, \frac{\varepsilon}{2}\right)
$$

for all sufficiently large $i \geq 1$ such that $X_{i}^{*} \leq(r+a) \gamma$.

Proof of (A.5). Note that $\boldsymbol{X}=\left\{\left|X_{i}^{*}-X_{j}^{*}\right|, i, j \geq 1, i<j\right\}$ is an additive process. Since $T^{(1) *} \cap \mathbb{R}$ is cycle-stationary (see, e.g. [29]), we have $\left\{\left|X_{i}^{*}-X_{j}^{*}\right|\right\} \stackrel{\mathrm{D}}{=}\left\{\left|X_{i+1}^{*}-X_{j+1}^{*}\right|\right\}$, where $0<\mathrm{E} X_{1}^{*}<\infty$. Thus, by the subadditive ergodic theorem (see [19, Theorem 1]), the finite limit $\lim _{i \rightarrow \infty} X_{i}^{*} / i=\zeta_{\boldsymbol{X}}$ exists $\mathrm{P}^{*}$-almost surely. Furthermore, consider the family $\boldsymbol{Y}=$ $\left\{Y_{i j}, i, j \geq 1, i<j\right\}$ with $Y_{i j}=c\left(X_{i}^{*}, X_{j}^{*}\right)$, where $c\left(X_{i}^{*}, X_{j}^{*}\right)$ denotes the shortest path length from $X_{i}^{*}$ to $X_{j}^{*}$ on $T^{(1) *}$. Then $\mathrm{E} Y_{01}=\mathrm{E} c\left(X_{0}^{*}, X_{1}^{*}\right)<\infty$ holds by condition (3.1) (see [32]), and we can again apply the subadditive ergodic theorem to show that the finite limit $\lim _{j \rightarrow \infty} c\left(X_{0}^{*}, X_{j}^{*}\right) / j=\zeta_{\boldsymbol{Y}}$ exists $\mathrm{P}^{*}$-almost surely. Furthermore, both $\boldsymbol{X}$ and $\boldsymbol{Y}$ are ergodic since $T$ is mixing; see [32]. Thus, $\zeta_{\boldsymbol{X}}$ and $\zeta_{\boldsymbol{Y}}$ are constant. Since $0<\mathrm{E} X_{1}^{*}=\zeta_{\boldsymbol{X}} \leq \zeta_{\boldsymbol{Y}}<\infty$, this gives

$$
\lim _{j \rightarrow \infty} \frac{c\left(o, X_{j}^{*}\right)}{X_{j}^{*}}=\lim _{j \rightarrow \infty} \frac{j}{X_{j}^{*}} \frac{c\left(X_{0}^{*}, X_{j}^{*}\right)}{j}=\xi,
$$

where $\xi=\zeta_{\boldsymbol{Y}} / \zeta_{\boldsymbol{X}} \in[1, \infty)$. Now let $\tilde{\varepsilon}>0$ such that $\tilde{\varepsilon}(r+a)<\varepsilon / 2$. Then (A.6) implies that $c\left(o, X_{i}^{*}\right) / X_{i}^{*}-\xi \in(-\tilde{\varepsilon}, \tilde{\varepsilon})$ with probability 1 for all sufficiently large $i$ and, therefore,

$$
\frac{1}{\gamma}\left(c\left(o, X_{i}^{*}\right)-\xi X_{i}^{*}\right) \in\left(-\frac{\varepsilon}{2}, \frac{\varepsilon}{2}\right)
$$

if $i$ is sufficiently large and $X_{i}^{*} / \gamma \leq r+a$.

Uniform integrability. Finally, we show that the family $\left\{\tilde{g}_{\gamma}, \gamma>0\right\}$ considered in (A.4) is uniformly $\left(v_{1} \otimes \mathrm{P}^{*}\right)$-integrable. From the ergodic theorem for stationary marked point processes (see [8, Theorem 12.2.IV]), we obtain

$$
\lim _{\gamma \rightarrow \infty} \frac{1}{\gamma} \sum_{\left\{X_{i} \in T^{(1)} \cap L^{+}:\left|X_{i}\right| \leq(r+1) \gamma\right\}} \frac{1}{\sin \alpha_{i}}=(r+1) \mathrm{E}\left(\sin \alpha^{*}\right)^{-1}
$$

almost surely and in $L^{1}$ since the point process $T^{(1)} \cap \mathbb{R}$ marked with the intersection angles is ergodic and $\mathrm{E}\left(\sin \alpha^{*}\right)^{-1}=\pi / 2<\infty$, where $\alpha^{*}$ denotes the typical intersection angle (see, e.g. [27, p. 288]). Using the inversion formula for Palm distributions of stationary marked point processes on $\mathbb{R}$ (see Proposition 11.3(iii) of [18]), this yields

$$
\mathbf{1}_{\left[0, X_{1}^{*}\right]}(x) \frac{1}{\gamma} \sum_{\left\{X_{i} \in\left(T^{(1) *}-x\right) \cap L^{+}:\left|X_{i}\right| \leq(r+1) \gamma\right\}} \frac{1}{\sin \alpha_{i}} \rightarrow(r+1) \mathbf{1}_{\left[0, X_{1}^{*}\right]}(x) \mathrm{E}\left(\sin \alpha^{*}\right)^{-1}
$$

in $L^{1}\left(v_{1} \otimes \mathrm{P}^{*}\right)$ as $\gamma \rightarrow \infty$. Furthermore, we have

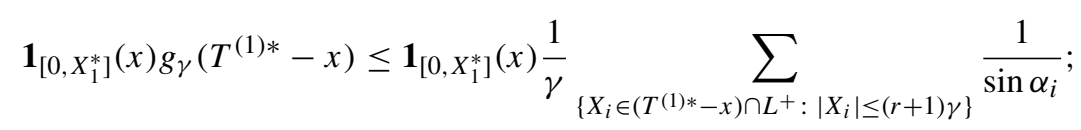

thus, the family $\left\{\tilde{g}_{\gamma}, \gamma>0\right\}$ considered in (A.4) is uniformly $\left(v_{1} \otimes \mathrm{P}^{*}\right)$-integrable. 


\section{Acknowledgement}

This work was supported by Orange Labs through research grant number 46143714.

\section{References}

[1] Aldous, D. J. and Kendall, W. S. (2008). Short-length routes in low-cost networks via Poisson line patterns. Adv. Appl. Prob. 40, 1-21.

[2] Baccelli, F. ANd Zuyev, S. (1996). Poisson-Voronoi spanning trees with applications to the optimization of communication networks. Operat. Res. 47, 619-631.

[3] Baccelli, F., Gloaguen, C. And Zuyev, S. (2000). Superposition of planar Voronoi tessellations. Commun. Statist. Stoch. Models 16, 69-98.

[4] Baccelli, F., Tchoumatchenko, K. And Zuyev, S. (2000). Markov paths on the Poisson-Delaunay graph with applications to routing in mobile networks. Adv. Appl. Prob. 32, 1-18.

[5] Baccelli, F., Klein, M., Lebourges, M. and Zuyev, S. (1997). Stochastic geometry and architecture of communication networks. Telecommun. Systems 7, 209-227.

[6] Bauer, H. (1981). Probability Theory and Elements of Measure Theory, 2nd edn. Academic Press, London.

[7] Daley, D. J. and Vere-Jones, D. (2003). An Introduction to the Theory of Point Processes, Vol. I, 2nd edn. Springer, New York.

[8] Daley, D. J. And Vere-Jones, D. (2008). An Introduction to the Theory of Point Processes, Vol. II, 2nd edn. Springer, New York.

[9] Fleischer, F., Gloaguen, C., Schmidt, V. and Voss, F. (2009). Simulation of the typical Poisson-VoronoiCox-Voronoi cell. J. Statist. Comput. Simul. 79, 939-957.

[10] Gloaguen, C., Voss, F. And Schmidt, V. (2009). Parametric distance distributions for fixed access network analysis and planning. In Proc. 21st Internat. Teletraffic Congress (Paris, September 2009), pp. 1-8.

[11] Gloaguen, C., Coupé, P., Maier, R. and Schmidt, V. (2002). Stochastic modelling of urban access networks. In Proc. 10th Internat. Telecommun. Network Strategy Planning Symp. (Munich, June 2002), VDE, Berlin, pp. 99-104.

[12] Gloaguen, C., Fleischer, F., Schmidt, H. and Schmidt, V. (2005). Simulation of typical Cox-Voronoi cells with a special regard to implementation tests. Math. Meth. Operat. Res. 62, 357-373.

[13] Gloaguen, C., Fleischer, F., Schmidt, H. and Schmidt, V. (2006). Fitting of stochastic telecommunication network models via distance measures and Monte-Carlo tests. Telecommun. Systems 31, 353-377.

[14] Gloaguen, C., Fleischer, F., Schmidt, H. and Schmidt, V. (2010). Analysis of shortest paths and subscriber line lengths in telecommunication access networks. Networks Spatial Econom. 10, 15-47.

[15] HaengGi, M. (2005). On distances in uniformly random networks. IEEE Trans. Inf. Theory 51, 3584-3586.

[16] HaEngGi, M. et al. (2009). Stochastic geometry and random graphs for the analysis and design of wireless networks. IEEE J. Sel. Areas Commun. 27, 1029-1046.

[17] Jensen, E. B. V. (1998). Local Stereology. World Scientific, River Edge, NJ.

[18] Kallenberg, O. (2002). Foundations of Modern Probability, 2nd edn. Springer, New York.

[19] Kingman, J. F. C. (1973). Subadditive ergodic theory. Ann. Prob. 1, 883-909.

[20] LACHIÈZE-Rey, R. (2009). Strong mixing property for STIT tessellations. Preprint. Available at http://arxiv.org/ abs/0905.1145v3.

[21] Lautensack, C. and Zuyev, S. (2008). Random Laguerre tessellations. Adv. Appl. Prob. 40, 630-650.

[22] Maier, R. and Schmidt, V. (2003). Stationary iterated tessellations. Adv. Appl. Prob. 35, 337-353.

[23] Matthes, K., Kerstan, J. And Mecke, J. (1978). Infinitely Divisible Point Processes. John Wiley, Chichester.

[24] NAGEL, W. AND WEISs, V. (2005). Crack STIT tessellations: characterization of stationary random tessellations stable with respect to iteration. Adv. Appl. Prob. 37, 859-883.

[25] OKabe, A., Boots, B., Sugihara, K. and Chiu, S. N. (2000). Spatial Tessellations: Concepts and Applications of Voronoi Diagrams, 2nd edn. John Wiley, Chichester.

[26] SchneIder, R. AND WeIL, W. (2008). Stochastic and Integral Geometry. Springer, Berlin.

[27] Stoyan, D., Kendall, W. S. And Mecke, J. (1995). Stochastic Geometry and Its Applications, 2nd edn. John Wiley, Chichester.

[28] Tchoumatchenko, K. and Zuyev, S. (2001). Aggregate and fractal tessellations. Prob. Theory Relat. Fields 121, 198-218.

[29] Thorisson, H. (2000). Coupling, Stationarity, and Regeneration. Springer, New York.

[30] Voss, F., Gloaguen, C. And Schmidt, V. (2009). Capacity distributions in spatial stochastic models for telecommunication networks. Image Anal. Stereology 28, 155-163.

[31] Voss, F., Gloaguen, C. And Schmidt V. (2009). Palm calculus for stationary Cox processes on iterated random tessellations. In Proc. 7th Internat. Symp. Modeling and Optimization of Mobile, Ad Hoc and Wireless Networks, IEEE Press, Piscataway, NJ, pp. 534-539. 
[32] Voss, F., Gloaguen, C. And Schmidt V. (2009). Scaling limits for shortest path lengths along the edges of stationary tessellations - Supplementary material. Preprint. Available at http://arxiv.org/abs/0912.4516v1.

[33] Voss, F., Gloaguen, C., Fleischer, F. and Schmidt, V. (2010). Densities of shortest path lengths in spatial stochastic networks. To appear in Stoch. Models.

[34] Voss, F., Gloaguen, C., Fleischer, F. and Schmidt, V. (2009). Distributional properties of Euclidean distances in wireless networks involving road systems. IEEE J. Sel. Areas Commun. 27, 1047-1055.

[35] Weiss, V. AND Nagel, W. (1999). Interdependences of directional quantities of planar tessellations. Adv. Appl. Prob. 31, 664-678.

[36] Zuyev, S. (1999). Stopping sets: gamma-type results and hitting properties. Adv. Appl. Prob. 31, 355-366.

[37] Zuyev, S. (2009). Stochastic geometry and telecommunications networks. In New Perspectives in Stochastic Geometry, eds W. S. Kendall and I. Molchanov, Oxford University Press, pp. 520-554 . 